\title{
Commentary
}

\section{The Role of Stromal Factors in Breast Tumorigenicity}

\author{
Danh Tran-Thanh, ${ }^{*}$ and Susan J. Done ${ }^{\star \dagger}$ \\ From the Department of Pathology, ${ }^{*}$ University Health Network, \\ Toronto, Ontario; and Departments of Laboratory Medicine and \\ Pathobiology and Medical Biophysics, ${ }^{\dagger}$ University of Toronto, \\ Toronto, Ontario, Canada
}

The importance of the relationship between tumor cells and adjacent stroma in breast carcinoma is increasingly recognized, but to date few factors involved in this interaction have been identified. In this issue of The American Journal of Pathology, de Neergaard et al ${ }^{1}$ demonstrate that EPST/1, an interferon response gene, can substitute for peritumoral fibroblasts and induce an epithelial-mesenchymal transition-like phenomenon in breast cancer cell lines.

When examined under the microscope, invasive breast carcinomas comprise, in addition to malignant epithelial cells, a considerable group of nonmalignant cells, including fibroblasts and myofibroblasts, blood and lymph vessels, and immune cells (including tumor infiltrating lymphocytes, macrophages, and mast cells), which reside within a remodeled interstitial stroma. The term tumor microenvironment has been widely used to describe both these surrounding stromal cells and the extracellular matrix in which they reside and is thought to be a product of a developing cross talk between epithelial tumor and stromal cells. In normal breast, the immediate area surrounding epithelial structures consists mainly of basement membrane, myofibroblasts, and an interstitial stroma. On invasion, malignant epithelial cells lose their contact with the basement membrane and are directly apposed to stromal fibroblasts and myofibroblasts. Indeed, in an early series of experiments, Ronnov-Jessen and Petersen ${ }^{2}$ demonstrated that when cocultured with tumor cells, normal fibroblasts underwent conversion to myofibroblasts, and in return these converted cells support tumor growth.

Previously, the tumor microenvironment was thought to act mainly as a support for tumor growth by providing sufficient nutrients via the production of new tumor blood vessels (angiogenesis), for example. More recently, however, the tumor microenvironment is being increasingly recognized as playing a major role in breast cancer progression and metastasis (reviewed in Weigelt and Bissel| ${ }^{3}$ ). Fibroblasts and myofibroblasts, which represent the major cell type in the tumor stroma of invasive breast carcinomas, are mainly derived from interstitial and perivascular fibroblasts, and there is evidence that they provide an essential communication network between the tumor and its stroma by secreting growth factors and cytokines. ${ }^{4}$ In experimental models, fibroblasts and mesenchymal cells have been demonstrated to increase the malignant properties of breast carcinoma cells lines, including invasiveness and enhanced motility. ${ }^{5}$ Thus, there is mounting evidence that the tumor microenvironment is of critical importance in providing the oncogenic signals required for cancer-cell proliferation and invasion, although the major players in this interaction are still being elucidated.

At the genetic level, there is also evidence supporting a relationship between the tumor and its microenvironment. Patocs et $\mathrm{al}^{6}$ demonstrated that stroma-specific loss of heterozygosity or allelic imbalance is associated with somatic TP53 mutations in neoplastic epithelial cells as well as lymph-node metastasis in patients with sporadic breast cancer. More recently, data from Trimboli et $\mathrm{al}^{7}$ suggested that Pten mutational status in mouse stromal fibroblasts can influence the initiation and development of breast epithelial tumors.

The clinical importance of the tumor microenvironment has been demonstrated by recent gene expression studies presenting specific "stroma-derived signatures" that can provide prognostic prediction of tumor behavior. Initial studies on the transcriptional alteration of fibroblasts cultured in serum led to the development of the so-called "wound response signature," a signature predictive of survival and metastasis in patients with breast cancer. ${ }^{8}$ Other profiles have since been published, and most are at least as accurate and some even outperform several published "epithelial-derived" gene expression signatures in predicting recurrence risk and survival. ${ }^{9}$

For tumors to progress and metastasize, they must acquire certain phenotypes that allow them to migrate to distant sites. These include, among other things, increased motility and invasiveness. But how do cancer cells acquire these traits? Recent evidence suggests the activation of embryonic transcription programs within cancer cells, which allow them to acquire properties of mesenchymal cells. This process, commonly known as

\footnotetext{
Accepted for publication December 4, 2009
}

Address reprint requests to Susan J. Done, MB, BChir, PhD, FRCPC, FCAP, FCCMG University Health Network, 200 Elizabeth Street, 11E444, Toronto, ON, M5G 2C4, Canada. E-mail: sdone@uhnres.utoronto.ca. 
epithelial-mesenchymal transition (EMT), is a phenotypic switch in which epithelial cells lose their characteristics and reorganize their cytoskeletal structure to acquire the properties and motility of mesenchymal cells. ${ }^{10}$ EMT is associated with changes in cell-cell adhesion, remodeling of the cell-matrix, and enhanced migratory capacity of tumor cells. There is increasing data that suggest the occurrence of EMT in tumor cells during the progression of certain types of cancers including breast cancer. ${ }^{10}$

In this issue of the AJP, de Neergaard et al. ${ }^{1}$ provide evidence that the tumor microenvironment is of critical importance in this process. They identify epithelial stromal interaction 1 (EPSTI1) as an important player in the interaction of tumor and adjacent stroma in breast cancer. EPST/1 is an interferon response gene originally identified in co-cultures of breast cancer cells with stromal fibroblasts. ${ }^{11}$ In an initial series of experiments, de Neergaard et al ${ }^{1}$ demonstrated that EPSTI1 can be induced both at the transcriptional and protein level in MDA-MD-231 breast cancer cell lines by exposure to interferon- $\alpha$ or co-cultivation with fibroblasts. Immunolocalization of EPSTI1 was identified in the nuclei and cytoplasm of treated cells. Additional immunostaining on a series of tissue samples showed EPSTI1 to be predominantly expressed in breast carcinomas as opposed to normal breast, and interestingly, the highest staining was found in breast cancer cells adjacent to stroma (epithelial-stromal junction) and in breast cancers with a basallike phenotype (as defined by cytokeratin 5 expression in this study). The latter subtype of breast cancer has recently been suggested to harbor increased numbers of tumor-initiating or cancer stem cells. ${ }^{12}$

In another pivotal set of experiments, de Neergaard et $\mathrm{al}^{1}$ demonstrated that EPSTI1 has the same effect as peritumoral-activated fibroblasts using a tumor environment assay. In the presence of fibroblasts, MCF-7 breast cancer cell line spread and invaded, forming irregular structures within a collagen gel matrix, whereas MCF-7 cells without co-cultured fibroblasts simply formed spherical clusters. Silencing EPSTI1 with small-interfering RNA before co-culture inhibited this spread. Interestingly, when stably transfected with EPST/1, MCF-7 cells autonomously spread and invaded, even without the presence of co-cultured fibroblasts. This led the authors to conclude that EPSTI1 can act as a substitute for fibroblasts in their assays. When examined for EMT characteristics, these transduced cells were shown to express the EMT markers fibronectin and $\alpha 2 \beta 1$ integrin, with reduced claudin-1 expression, a marker reputed to be low in stem-cell like breast cancer cells. However, levels of E-cadherin, an epithelial marker, still remained unaltered. Moreover, RT-PCR analysis of the transfected cells showed upregulation of putative EMT-inducing transcription factors SLUG and TWIST. In addition, phenotypically, EPSTI1 expressing MCF-7 cells had more spindle-cell morphology and demonstrated increased migration using a Boyden-chamber transwell assay. Collectively, the data obtained led the authors to conclude that EPSTI1 may be a mechanism by which peritumoral fibroblasts induce EMT-like changes in tumor epithelial cells (albeit admit- tedly incomplete because no change in E-cadherin level was observed).

The microenvironment of cancer cells is thought to provide the necessary signals to induce transcription factors, and it is the stroma that stimulates the necessary mesenchymal phenotype in cancer cells required to invade and migrate to distant tissues. Although increasingly recognized, few factors have been demonstrated to be directly involved in this interaction. The data provided by de Neergaard et $\mathrm{al}^{1}$ in this issue adds weight to the current hypothesis whereby, through the secretion of growth factors and cytokines (interferon, in the case of EPSTI1), fibroblasts can induce proliferative and transformative signals in adjacent epithelial cells.

Another interesting finding in this report is that transfected cells that expressed EPSTI1 demonstrated increased mammosphere forming ability, a property usually restricted to stem cells. In addition, some induction of putative stem cell markers was demonstrated with EPSTI1 expression. Recently, data from Mani et al ${ }^{13}$ showed that EMT confers tumor cells with some cancer stem cell properties and that, conversely, cancer stem cells express EMT markers. By definition, cancer stem cells refer to a small subset of cancer cells that can initiate tumorigenesis by undergoing self-renewal and differentiation, whereas the remaining majority of cells cannot. ${ }^{14}$ For example, a small subpopulation of cancer cells in some human breast tumors exhibit a CD44high/ CD24 ${ }^{\text {low }}$ phenotype; these are highly enriched for tumorinitiating cells that in limiting dilution transplantation will generate tumors in a xenograft model, in comparison with the majority of cancer cells with a CD $44^{\text {low }} / C D 24^{\text {high }}$ phenotype found in the same tumor. ${ }^{15}$

In their experiments, de Neergaard et $\mathrm{al}^{1}$ demonstrated that silencing EPST/1 via small-interfering RNA in MDA-MD-231cells decreased their tumor forming capacity when inoculated into NOD/SCID mice. Although the authors do not evaluate the "stemness" of their EPSTI1transduced MCF-7 cells beyond that of mammosphere formation, this remains an important and exciting finding whereby there may be a direct relationship between EMT and cancer stem cells. It is, therefore, tempting to speculate that EMT inducers, ie, cancer-associated fibroblasts and other stromal cells, can act as a niche for stem cells and modulate tumor evolution by transiently modifying the stem cell composition of tumors.

The concept that the tumor microenvironment can induce cancer stem cells via an EMT process is appealing and thus warrants further investigation. This will undoubtedly lead to better insights into cancer biology and has the potential to result in a new class of therapeutic agents. Attacking both the tumor cells and their supporting and sustaining stroma may be more effective than targeting the carcinoma alone and could become a standard approach.

\section{References}

1. de Neergaard M, Kim J, Villadsen R, Fridriksdottir AJ, Rank F Timmermans-Wielenga V, Langerød A, Børresen-Dale A-L, Petersen 
O-W, Rønnov-Jessen L: Epithelial-Stromal Interaction 1 (EPSTI1) substitutes for peritumoral fibroblasts in the tumor microenvironment. Am J Pathol, 176:1229-1240

2. Ronnov-Jessen L, Petersen OW: Induction of alpha-smooth muscle actin by transforming growth factor-beta 1 in quiescent human breast gland fibroblasts: implications for myofibroblast generation in breast neoplasia. Lab Invest 1993, 68:696-707

3. Weigelt B, Bissell MJ: Unraveling the microenvironmental influences on the normal mammary gland and breast cancer. Semin Cancer Biol 2008, 18:311-321

4. Kalluri R, Zeisberg M: Fibroblasts in cancer. Nat Rev Cancer 2006, 6:392-401

5. Karnoub AE, Dash AB, Vo AP, Sullivan A, Brooks MW, Bell GW, Richardson AL, Polyak K, Tubo R, Weinberg RA: Mesenchymal stem cells within tumour stroma promote breast cancer metastasis. Nature 2007, 449:557-563

6. Patocs A, Zhang L, Xu Y, Weber F, Caldes T, Mutter GL, Platzer P, Eng C: Breast-cancer stromal cells with TP53 mutations and nodal metastases. N Engl J Med 2007, 357:2543-2551

7. Trimboli AJ, Cantemir-Stone CZ, Li F, Wallace JA, Merchant A, Creasap N, Thompson JC, Caserta E, Wang H, Chong JL, Naidu S, Wei G, Sharma SM, Stephens JA, Fernandez SA, Gurcan MN, Weinstein MB, Barsky SH, Yee L, Rosol TJ, Stromberg PC, Robinson ML, Pepin F, Hallett M, Park M, Ostrowski MC, Leone G: Pten in stromal fibroblasts suppresses mammary epithelial tumours. Nature 2009, 461:1084-1091

8. Chang HY, Sneddon JB, Alizadeh AA, Sood R, West RB, Montgomery K, Chi JT, van de Rijn M, Botstein D, Brown PO: Gene expression signature of fibroblast serum response predicts human cancer progression: similarities between tumors and wounds. PLoS Biol 2004, 2:E7

9. Finak G, Bertos N, Pepin F, Sadekova S, Souleimanova M, Zhao H, Chen H, Omeroglu G, Meterissian S, Omeroglu A, Hallett M, Park M Stromal gene expression predicts clinical outcome in breast cancer. Nat Med 2008, 14:518-527

10. Polyak K, Weinberg RA: Transitions between epithelial and mesenchymal states: acquisition of malignant and stem cell traits. Nat Rev Cancer 2009, 9:265-273

11. Nielsen HL, Ronnov-Jessen L, Villadsen R, Petersen OW: Identification of EPSTI1, a novel gene induced by epithelial-stromal interaction in human breast cancer. Genomics 2002, 79:703-710

12. Honeth G, Bendahl PO, Ringner M, Saal LH, Gruvberger-Saal SK, Lovgren K, Grabau D, Ferno M, Borg A, Hegardt C: The CD44+/ CD24- phenotype is enriched in basal-like breast tumors. Breast Cancer Res 2008, 10:R53

13. Mani SA, Guo W, Liao MJ, Eaton EN, Ayyanan A, Zhou AY, Brooks M, Reinhard F, Zhang CC, Shipitsin M, Campbell LL, Polyak K, Brisken C, Yang J, Weinberg RA: The epithelial-mesenchymal transition generates cells with properties of stem cells. Cell 2008, 133:704-715

14. Marotta LL, Polyak K: Cancer stem cells: a model in the making. Curr Opin Genet Dev 2009, 19:44-50

15. Al-Hajj M, Wicha MS, Benito-Hernandez A, Morrison SJ, Clarke MF: Prospective identification of tumorigenic breast cancer cells. Proc Natl Acad Sci USA 2003, 100:3983-3988 